\title{
Erratum: Asymptotic freedom at zero temperature in free-standing crystalline membranes [Phys. Rev. B 89, 125433 (2014)]
}

\author{
E. I. Kats and V. V. Lebedev \\ (Received 29 July 2016; published 12 August 2016)
}

DOI: 10.1103/PhysRevB.94.079904

In our paper we missed the sign in the one-loop renormalization-group (RG) equation for the bending modulus $\kappa$. With the correct sign (plus) Eq. (11) is

$$
\frac{d \kappa}{d \xi}=+\frac{\hbar}{8 \pi \rho^{1 / 2} \kappa^{1 / 2}} \frac{3 \mu^{2}+3 \mu \lambda}{2 \mu+\lambda} .
$$

Here the coefficients $\mu$ and $\lambda$ are $2 d$ Lame (elastic) moduli, and $\kappa$ is the Helfrich (bending) module of the membrane. A corresponding change in sign should be performed in the Eq. (13) derived form of Eq. (11) near the fixed point $\lambda=-\mu / 2$,

$$
\frac{d \kappa}{d \xi}=+\frac{\hbar \mu}{8 \pi \rho^{1 / 2} \kappa^{1 / 2}},
$$

and introducing the dimensionless coupling constant $g$ one finds

$$
\frac{d g}{d \xi}=-g^{2}, \quad g=\frac{5 \hbar \mu}{32 \pi \rho^{1 / 2} \kappa^{3 / 2}} .
$$

Its solution evidently decreases as the scale grows

$$
g=g_{0} /\left(1+g_{0} \xi\right)
$$

Although the methodology of the paper, all expressions for the renormalization of the effective action presented in the Appendix, and RG equations for the Lame coefficients remain correct, we were mistaken in the claim that the quantum fluctuations destabilize the flat state of the free-standing crystalline membrane leading to the asymptotic freedom regime. The correct situation is just the opposite. With the correct sign in the one-loop equation (11), bending oscillations become harder due to the quantum fluctuations (similar to thermal fluctuations which lead to hardening the oscillations). Very recently the authors of Ref. [1] also found stabilization of the flat membrane phase in quantum polymerized membranes.

We would like to thank I. S. Burmistrov, I. V. Gornyi, V. Y. Kachorovskii, and A. D. Mirlin, who, working in the same field, let us know (prior to their publication) that they found that quantum fluctuations stabilize the flat state of a free-standing crystalline film.

[1] O. Coquand and D. Mouhana, The flat phase of quantum polymerized membranes, arXiv:1607.03335. 